\title{
EDITORIAL
}

\section{The Planning, Organization, Management and Quality Assurance of Health Care Systems}

The Planning of the "Health Care Systems" (HCS) of a country is aimed to meet the health care needs of the society. The HCS have three dimensions, (i) length, (ii) width and (iii) depth. The length must be large enough to ensure even access to all sections of population (rich, poor, urban, rural). The width is comprised of all specialties that are required to address the types of the diseases and health problems of the society. The depth is determined by the technological advancements being practiced in delivering the health care, and is based on the available resources.

The Organization of the HCS is based upon three (3) major pillars, (i) The Health Care Delivery Organizations (HCDO)/hospitals, (ii) The Health Care Providers (HCP), and (iii) The Health Care Services. The number and technical abilities of HCDO and HCP are based upon the size of the population and the types of the health care problems of the society. On the basis of the technical level of health care, the HCDO are further categorized into specialized, tertiary, secondary and primary health care delivery organizations; and are further divided into three types, (i) the curative HCDO, (ii) preventive HCDO, and (iii) combined curative and preventive HCDO. The HCP are comprised of medical, nursing, allied health and paramedical workforce. The HCP have three essential elements, (i) How they are trained, (ii) How their health care practices are regulated, and (iii) How they are paid. The Health Care Services are organized in accordance to the prevalence and pattern of the diseases and health care problems faced by the society.

The Management of the HCS is carried out, (i) at government level by political leaders and civil servants, (ii) at HCDO level by the autonomous corporate management comprising of all stake holders including representatives of the HCP, and (iii) at department or service delivery level by the HCP alone. According to the modern thought of public policy, the laws, rules, regulations and policies are developed by the government with support of the stake holders. At the HCDO level, the institutional regulations and policies are developed by the corporate management. At department or service delivery level the HCP are responsible to lay down and carry out the written protocols and procedures that are also known as health care standards. The effectiveness of the standards is measured to determine the quality.

The Quality Assurance is achieved by reviewing the health care services at three (3) steps; (i) the continuous monitoring is performed by the leader of the clinical service unit and is reported at end of each calendar year, (ii) the internal review of each specialty health care services is performed by two peers (professionals) of the same specialty after every two to three years, and (iii) the external review or accreditation is performed by a third party after every five to ten years.

In brief, the "Health Care Systems" are planned in light of (i) size of the population, (ii) prevalence of the diseases, and (iii) available resources; are organized on the basis of health care; (i) organizations, (ii) providers, and (iii) services; and are managed through laws, rules, regulations, policies, protocols, procedures by the (i) government, (ii) autonomous corporate managements of HCDO, and (iii) HCP. The quality assurance of the health care services is carried out by (i) continuous monitoring, (ii) internal review, and (iii) accreditation.

It is hoped that the readers of Annals of KEMU will find this description useful while considering "Health Care Systems” at National, Provincial, Districts or Institutional level.

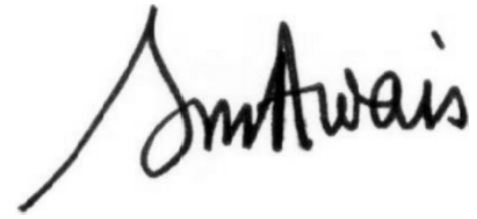

Prof. Dr. Syed Muhammad Awais

(Sitara-e-Imtiaz)

Editor 